\title{
EQUIDADE NO USO DE SERVIÇOS ODONTOLÓGICOS ENTRE ADOLESCENTES BRASILEIROS: UMA ANÁLISE MULTINÍVEL
}

\section{EQUITY IN THE USE OF DENTAL SERVICES BETWEEN ADOLESCENTS BRAZILIANS: A MULTILEVEL ANALYSIS}

\author{
Renata Francine Oliveira \\ Universidade Estadual de Montes Claros \\ renatafrancine@gmail.com \\ Desirée Sant Ana Haikal \\ Universidade Estadual de Montes Claros \\ desireehaikal@gmail.com
}

Danilo Lima Carreiro Instituto Federal do Norte de Minas-IFNMG danilolimacarreiro@gmail.com

Marise Fagundes Silveira Universidade Estadual de Montes Claros ciaestatistica@yahoo.com.br

Andréa Maria Eleutério de Barros Lima Martins Universidade Estadual de Montes Claros martins.andreamebl@gmail.com

\begin{abstract}
RESUMO
O estudo teve por objetivo avaliar se o uso dos serviços odontológicos no SUS entre adolescentes brasileiros favorece a equidade. Foram utilizados dados de 4654 adolescentes (15-19 anos) participantes do levantamento SB Brasil 2010 e dados contextuais relativos à municípios. A variável dependente foi o tipo de serviço odontológico utilizado (SUS/ Outros serviços). Regressão logística multinível foi realizada. A prevalência do uso dos serviços odontológicos no SUS foi de $46,8 \%$. O uso do SUS mostrou-se associado aos determinantes contextuais IDHM e média de escovação dental, e aos fatores individuais anos de estudo, renda, cor da pele, tempo e motivo do uso, presença de dentes cariados, presença de dentes perdidos, satisfação com dentes e boca e avaliação da consulta. Condições mais vulneráveis foram associadas ao maior uso no SUS. Assim, a equidade vem sendo alcançada no uso de serviços odontológicos no SUS.
\end{abstract}

Palavras-Chave: Assistência odontológica. Sistema Único de Saúde. Comportamento do Adolescente. Equidade.

\begin{abstract}
The objective of the study was to evaluate whether the use of dental services at SUS among Brazilian adolescents favors the equity. Data were used for adolescents 15 to 19 years participants of the survey SB Brazil 2010 and contextual data relating to municipalities of databases, public. The dependent variable was: SUS and other services. Multilevel logistic regression was performed with 4654 adolescents. The prevalence of the use of dental services in the SUS was $46.8 \%$. The contextual determinants were IDHM and average brushing. Individual factors were: years of study, income, skin color, time, reason for use, decayed teeth and lost, besides satisfaction with teeth and mouth and assessment of the consultation. The most vulnerable conditions were associated to higher use in SUS. The fairness is being achieved in the use of dental services in the SUS.
\end{abstract}

Keywords: Dental Care. Health System. Adolescent Behavior. Equity.

Recebido em: 27/02/2017

Aceito para publicação em: 27/02/2018 


\section{INTRODUÇÃO}

O quadro epidemiológico da saúde bucal da população jovem brasileira melhorou nas últimas décadas (Narvai,1999; Marcenes, 2001). A redução dos índices de cárie ocorreu por causa de mudanças nos critérios de diagnóstico, melhoria das condições socioeconômicas das pessoas, uso do flúor de forma preventiva e por políticas de saúde que incluíam programas preventivos e curativos para a população jovem (Nickel, Lima e Silva, 2008; Peres et al, 2012; Okunseri et al, 2013). Porém apesar deste declínio observa-se também uma polarização da doença nos grupos menos privilegiados (Peres et al, 2012; Okunseri et al, 2013). Iniquidades relacionadas ao nível individual como a raça/cor da pele autodeclarada, renda, escolaridade e residência em zona rural já foram associadas à doenças bucais (Frias et al, 2007; Claro et al, 2006). Além deles, fatores contextuais, ou seja, referentes ao município e/ou ao local de residência das pessoas, também foram associados à cárie; dentre eles o maior acesso a esgoto doméstico e os índices de exclusão social (Frias et al, 2007).

A criação do sistema público de saúde brasileiro (SUS), em 1988, gerou uma complexa rede de serviços de saúde, com o objetivo de prover a atenção em saúde sob os princípios da universalidade, equidade e integralidade (Paim, 2011). Universalidade é garantir o direito à saúde para todas as pessoas. A equidade se manifesta na existência de um sistema público onde os serviços de saúde sejam distribuídos segundo o perfil de necessidades de cada grupo populacional, visando maior igualdade (WHO, 2010; Travassos, 1997; Brasil, 1990). Já a integralidade, segundo Brasil (2004), é entendida como o conjunto articulado e contínuo das ações e serviços preventivos e curativos, individuais e coletivos, exigidos para cada caso, em todos os níveis de complexidade do sistema. Para De Matos (2011), a mudança das práticas do SUS pautadas por estes conceitos abriria à perspectiva de se reverter a imagem simbólica de que a saúde privada ou suplementar seja de qualidade superior à pública.

Os serviços públicos de saúde devem ofertar ações de promoção à saúde, prevenção dos fatores de risco, assistência aos danos e reabilitação segundo a dinâmica do processo saúde-doença, e estas devem estar integradas em todos os espaços organizacionais do sistema de saúde (Machado et al 2007). Por décadas, ocorreu a priorização de crianças e adolescentes ao acesso à assistência odontológica. Entretanto, a criação da Estratégia Saúde da Família (ESF) com inserção da equipe de saúde bucal promoveram mudanças nos critérios de acesso e utilização dos serviços odontológicos. Adultos, gestantes e idosos passaram a ser também considerados no que diz respeito à assistência odontológica (Costa et al 2006; Brasil, 2006).

Estudos que avaliem se estão sendo alcançados os princípios da universalidade, da equidade e da integralidade propostos pelo SUS são necessários, uma vez que colocar em prática tais princípios permanece como desafio para o SUS. Entretanto, estudos da distribuição, disponibilidade e cobertura dos serviços odontológicos com representatividade nacional são escassos, apesar de sua relevância. Um estudo que comparou o uso de serviços entre adolescentes, adultos e idosos apontou que, dentre os três grupos, os adolescentes apresentaram maior prevalência de uso no SUS Brasil (Brasil, 1990; Borges et al 2010; Peres et al 2012). Outro estudo conduzido entre adolescentes que viviam em território da estratégia saúde da família encontrou maior uso do serviço odontológico público do que privado (Claro et al, 2006).

Segundo Frias, et al (2006), os serviços de saúde necessitam de informações específicas sobre cada segmento populacional, referentes às suas maiores necessidades, a fim de direcionar recursos e investimentos para as áreas com maior demanda. A compreensão da relação complexa entre o uso dos serviços e seus determinantes é feita considerando modelos teóricos explicativos sobre o uso de serviços de saúde e determinantes sociais da saúde (Pavão e Coeli, 2008). Nesse sentido, propõe-se a utilização de um modelo teórico para analisar se a equidade tem sido alcançada no uso dos serviços odontológicos no SUS entre adolescentes brasileiros considerando questões contextuais e individuais.

\section{METODOLOGIA}

Trata-se de um estudo transversal de prevalência analítico, que considerou variáveis contextuais e individuais. O modelo teórico proposto foi baseado em informações do contexto referente aos determinantes sociais de saúde e em modelos teóricos prévios sobre uso de serviços que utilizam 
informações individuais objetivas, subjetivas e também de qualidade dos serviços odontológicos (Figura 1) (Solar \& Irwin, 2007; Davidson \& Andersen, 1997; Gift et al, 1998; Donabedian, 1998).

Foi utilizada abordagem multinível, que leva em considerações variáveis contextuais (nível municipal) e individuais, para buscar explicar um desfecho medido no nível individual.

Para o nível contextual, foram coletados dados secundários em sua maioria do ano de 2010 , de bases de dados públicos do Departamento de Informática do SUS (DATA/SUS/MS), Instituto Brasileiro de Geografia e Estatística (IBGE) e Atlas Brasil 2013 do Programa das Nações Unidas para o Desenvolvimento - PNUD; da Pesquisa Nacional de Saneamento Básico do IBGE realizada em 2008, considerando os 177 municípios avaliados pelo SB Brasil 2010 (IBGE, 2011; Brasil, 2009 Roncalli et al, 2012). O Atlas Brasil e a Pesquisa Nacional de Saneamento Básico são bases de dados geradas do censo demográfico de 2010 (coleta de dados entre $1^{\circ}$ de agosto e 30 de outubro de 2010, utilizando a base territorial de 316.574 setores censitários) (Brasil, 2009).

No nível individual, utilizou-se os dados do inquérito epidemiológico nacional de saúde bucal, o SB Brasil 2010 (Roncalli et al, 2012) . Neste inquérito foram entrevistadas e examinadas pessoas de 177 municípios, incluindo as 27 capitais dos estados brasileiros, todos selecionados por amostragem probabilística por conglomerados em múltiplos estágios, com probabilidade proporcional ao tamanho e considerando um efeito de desenho (deff) igual a 2 (Roncalli et al, 2012). Os exames e entrevistas foram realizados sob luz natural, por cirurgiões-dentistas previamente treinados e calibrados pela técnica do consenso, sendo o valor mínimo aceitável de kappa para cada examinador, grupo etário e agravo estudado igual a 0,65 . As entrevistas foram realizadas com auxílio de computador de mão (Personal Digital Assistant) (Roncalli et al, 2012). Neste estudo, foram considerados os dados relativos aos adolescentes de 15-19 anos participantes do SB Brasil 2010. Os indivíduos que não usaram serviços odontológicos, ou não responderam a pergunta que originou a variável dependente, foram excluídos das análises.

A variável dependente foi o tipo de serviço odontológico utilizado, referindo-se ao local de uso dos serviços odontológicos (público, privado, planos de saúde, outros). Ela foi coletada através da seguinte pergunta: "Onde foi a sua última consulta (odontológica)?". Posteriormente as opções de resposta foram categorizadas de forma dicotômica em "SUS" versus "Outros".

As variáveis independentes foram reunidas em subgrupos, conforme modelo teórico adotado (Figura 1). O modelo levou em conta Variáveis de Contexto, Determinantes Primários Individuais, Comportamentos em Saúde, Condições de Saúde Bucal e Avaliação do Serviço Utilizado.

\section{Variáveis independentes contextuais}

As variáveis contextuais, em sua maioria, referentes ao ano 2010, a fim de haver coerência com o ano de coleta dos dados individuais. Foram consideradas informações sobre Ambiente, Condição social/econômica, Informações do Sistema de Saúde e Indicadores contextuais de assistência. A variável contextual obtida do Censo Demográfico de 2010 do IBGE foi referente ao tipo de Município (capital/interior). As contextuais obtidas do Atlas Brasil foram: acesso a banheiro e água encanada tratada ( $\geq$ média $83,02 \%$ / < média $83,02 \%$ ); acesso à coleta de lixo ( $\geq$ média $95,86 \%$ / < média 95,86\%); Índice de Desenvolvimento Humano Municipal - IDHM - $(\geq 0,7 /<0,7)$; Índice de GINI $(\geq 0,59$ / $<0,59)$. A variável contextual da Pesquisa Nacional de Saneamento Básico do IBGE, realizada em 2008, foi referente à fluoretação no município (fluoretado/ não fluoretado).

As variáveis contextuais referentes às políticas de saúde bucal foram: média do número de cirurgiões dentistas por 1000 habitantes no município ( $\geq$ média $0,66 /<$ média 0,66 ); média do número de cirurgiões dentistas do SUS por 1000 habitantes no município ( $\geq$ média $0,40 /<$ média 0,40 ); cobertura de equipes de saúde bucal na atenção primária ( $\geq$ meta $40 \% /<$ meta $40 \%$ ); presença de Centro de Especialidades Odontológicas - CEO no município (presença/ausência) (Brasil2010). As variáveis contextuais referentes Indicadores de assistência incluiu a média de escovação supervisionada no ano ( $\geq$ que a meta de $3 \% /$ < que a meta de 3\%) e o "Índice de cuidados odontológicos". Essa última foi construída a partir de dados agregados do inquérito SB Brasil, tendo sido calculada pela proporção da soma dos dentes restaurados sobre a soma do total de dentes cariados, perdidos e restaurados - CPOD. Tratase de uma medida que possibilita comparações entre diferentes populações ao longo do tempo (Roncalli et al, 2012). Esta variável foi categorizada considerando valores acima e abaixo da média dos valores obtidos nos 177 municípios brasileiros participantes do SB Brasil 2010. 


\section{Variáveis independentes individuais}

Os Determinantes Primários Individuais incluíram: idade em anos ( $\geq 15<18$ anos/ $\geq 18<19$ anos), anos de estudo ( $\geq 12 / 9-11 / 5-8 / 0-4)$, renda média familiar em reais $(\geq 1500,00 /<1500,00)$, sexo, cor da pele autodeclarada (dicotomizada em brancos / negros-pardosamarelos-indígenas) e os Comportamentos em Saúde compreenderam as variáveis relacionadas ao tempo desde a última consulta em anos $(\leq 1 />1)$ e ao motivo da consulta (revisão-prevenção/ dor-tratamento-extraçãooutros).

As variáveis referentes às Condições de Saúde Bucal foram divididas em normativas e subjetivas. As normativas consideradas foram: presença de dentes cariados $(0 / \geq 1)$, presença de dentes perdidos $(0 / \geq 1)$, presença de dentes restaurados $(0 / \geq 1)$, presença de oclusopatias (Não/sim, obtida através do Indice de estética dental que é uma combinação de 11 medidas considerando dentição, estética e oclusão e que define necessidade de tratamento de oclusopatias). As variáveis subjetivas consideradas foram: dor de dente (Não/ Sim); autopercepção da necessidade de tratamento (Não/ Sim); autopercepção da necessidade de prótese (Não/ Sim), satisfação com dentes e boca (satisfeito/ indiferente-insatisfeito), o impacto das desordens bucais no desempenho diário (OIDP) (Não/ Sim).

A Avaliação do Serviço Usado foi medida pela variável avaliação da consulta (dicotomizada em Muito bom-bom/ Regular-Ruim-/Péssimo (Sussex, 2010)).

Os dados foram organizados inicialmente no Statistical Package for the Social Sciences ${ }^{\circledR}$ (SPSS 19.0) para a realização das análises descritivas e bivariadas. As análises descritivas das variáveis independentes individuais, em função do efeito do desenho amostral complexo, utilizaram o comando Complex Samples do programa SPSS. Em seguida, foram conduzidas análises bivariadas pelo Teste Qui-quadrado e foram consideradas nos modelos múltiplos somente as variáveis individuais que apresentaram nível de significância (valor-p) igual ou inferior a 0,20 em tais análises. Já com relação as variáveis de contexto, todas foram inicialmente incluídas e testadas nos modelos múltiplos. Um mapa que demonstra o uso de serviços odontológicos no SUS e o efeito da diferença dos contextos foi construído com base na representatividade das macrorregiões e capitais garantidas no estudo. $O$ modelo múltiplo foi composto de forma multinível (hierárquica) através da Regressão Logistica Binária.

A análise multinível utilizou o modelo de efeitos fixos (modelo de intercepto) usando a família mixed para rodar (Snijders, 2011), para a estimação do ajuste entre o desfecho (uso de serviços no SUS) e as variáveis explicativas de primeiro (cidades participantes) e segundo (indivíduos examinados) níveis da análise com os coeficientes mistos e função logit para obtenção das medidas de odds ratios (OR) e IC $95 \%$ (Tabela 2). O programa STATA ${ }^{\otimes}$ v 14.0 foi usado nessa etapa da análise. O primeiro modelo (modelo 1) contém apenas a variável dependente, sem as demais variáveis. O Modelo 2 apresenta todas as variáveis contextuais, ainda não ajustado. $O$ modelo 3 , partiu do ajuste do modelo 2 , e nele foram adicionadas as variáveis individuais que tiveram valor $p<0,20$ na análise bivariada. $A$ Tabela 3 apresenta os modelos ajustados apenas com as variáveis contextuais (Modelo 4) e o modelo final ajustado com as variáveis significantes (Modelo 5). O Deviance foi o indicador usado para avaliar a medida de qualidade do ajuste, possibilitando comparar as funções de verossimilhança, e é representado pelo -2loglikelihood. Foi observada a redução na variabilidade do efeito aleatório, pela comparação com o modelo anterior considerando como melhor ajuste o modelo que reduziu valor maior que a distribuição chi $^{2}$ para o número de grau de liberdade definido pelo número de variáveis incluídas no modelo. Ao final, foram mantidas no modelo somente as variáveis que permaneceram associadas ao desfecho ao nível de $5 \%(\alpha=0,05)$.

O levantamento epidemiológico seguiu as diretrizes nacionais e internacionais de ética em pesquisas envolvendo seres humanos, tendo recebido aprovação do Conselho Nacional de Ética (Processo CONEP 009/2010, registro 15498). 
Figura 1 - Modelo Teórico de avaliação dos determinantes do uso de serviços odontológicos públicos

\begin{tabular}{|c|c|c|c|c|}
\hline $\begin{array}{c}\text { VARIÁVEIS } \\
\text { DE } \\
\text { CONTEXTO }\end{array}$ & $\begin{array}{c}\text { DETERMINANTES } \\
\text { PRIMÁRIOS } \\
\text { INDIVIDUAIS }\end{array}$ & COMPORTAMENTOS EM SAÚDE & CONDIÇŌES DE SAÚDE & AVALIAÇÁO DO SERVIÇO USADO \\
\hline $\begin{array}{l}\text { Ambiente } \\
\text { Tipo de município (capital/interior) } \\
\text { Acesso a água banheiro e agua tratada no } \\
\text { domicílio } \\
\text { Acesso à Coleta de lixo } \\
\text { Fluoretação de água }\end{array}$ & $\begin{array}{l}\text { Características Pessoais } \\
\text { Imutáveis } \\
\text { Sexo } \\
\text { Idade } \\
\text { Raça/Cor da pele } \\
\text { autodeclarada }\end{array}$ & $\begin{array}{l}\text { Uso Formal dos Serviços Odontológicos } \\
\text { Sim /não } \\
\text { Periodicidade do uso quanto ao tempo }\end{array}$ & & \\
\hline $\begin{array}{l}\text { Condição Social / Econômica } \\
\text { IDH } \\
\text { Îndice de GINI }\end{array}$ & & $\begin{array}{l}\text { Motivo do uso } \\
\text { Rotina } \\
\text { Prevenção } \\
\text { Manutenção de tratamento } \\
\text { Urgência }\end{array}$ & $\begin{array}{l}\text { Condição Saúde Bucal } \\
\text { Normativa/ objetiva } \\
\text { Presença de dentes cariados Presença } \\
\text { de dentes perdidos } \\
\text { Presença de dentes restaurados } \\
\text { Presença de Oclusopatias }\end{array}$ & $\begin{array}{l}\text { Avaliação do serviço } \\
\text { Na perspectiva dos usuários } \\
\text { Avaliaçãa da consulta }\end{array}$ \\
\hline & & & $\begin{array}{l}\text { Subjetiva } \\
\text { Dor de Dente } \\
\text { Autopercepção da necessidade } \\
\text { de tratamento } \\
\text { Autopercepção da saúde bucal } \\
\text { Satisfação com a saúde bucal } \\
\text { Autopercepção da necessidade de } \\
\text { prótese } \\
\text { Impactos das desordens bucais }\end{array}$ & \\
\hline $\begin{array}{l}\text { Sistema de Saúde } \\
\text { Politica de saúde bucal } \\
\text { Média Nº CDs por } 1000 \text { hab } \\
\text { MédiaN }{ }^{\circ} \text { CDs SUS por } 1000 \text { hab } \\
\text { Cobertura Saúde Bucal na Atenção } \\
\text { Primária } \\
\text { Presença de CEO }\end{array}$ & $\begin{array}{l}\text { Características Pessoais } \\
\quad \text { Mutáveis } \\
\text { Escolaridade } \\
\text { Renda }\end{array}$ & $\begin{array}{l}\text { Tipo de serviço utilizado } \\
\text { Público / Governamental / SUS } \\
\text { Filantrópico } \\
\text { Supletivo } \\
\text { Privado }\end{array}$ & & \\
\hline $\begin{array}{l}\text { Indicadores de Assistência } \\
\text { Média de escovação supervisionada } \\
\text { Índice de Cuidados Odontológicos }\end{array}$ & & & & \\
\hline
\end{tabular}




\section{RESULTADOS}

Dos 5445 adolescentes investigados no SB Brasil 2010, 4654 foram considerados neste estudo, pois, 791 preencheram os critérios de exclusão. Um total de $726(13,3 \%)$ adolescentes nunca havia consultado o dentista na vida. As informações contextuais dos municípios avaliados no inquérito mostraram que a maioria dos indivíduos vivia no interior $(66,9 \%)$, tinha banheiro e água encanada tratada $(69,6 \%)$ e coleta de lixo (73,8\%), e moravam em locais que em sua maioria ofereciam água fluoretada (84,3\%).

A maioria dos indivíduos vivia em municípios que apresentavam IDHM alto $(80,3 \%)$, índice de Gini menor que o resultado nacional de $0,59(63,0 \%)$, cuja proporção de cirurgiões-dentistas no SUS era menor que a média nacional $(63,6 \%)$. A maior porcentagem de indivíduos vivia em municípios que não executavam escovação supervisionada conforme a meta nacional sugerida para $2010(74,4 \%)$ e em locais que não atingiram a proporção de $40 \%$ de cobertura de equipes de saúde bucal na estratégia saúde da família $(68,8 \%)$.

A Figura 2 apresenta o mapa a prevalência de uso dos serviços odontológicos no SUS entre os adolescentes, segundo cada macrorregião e cada capital participantes do inquérito epidemiológico demonstrando a variabilidade desta frequência em cada contexto.

Figura 2 - Representação do uso de serviços por adolescentes no SUS nas macrorregiões e capitais brasileiras com base nos dados do SB Brasil, 2010

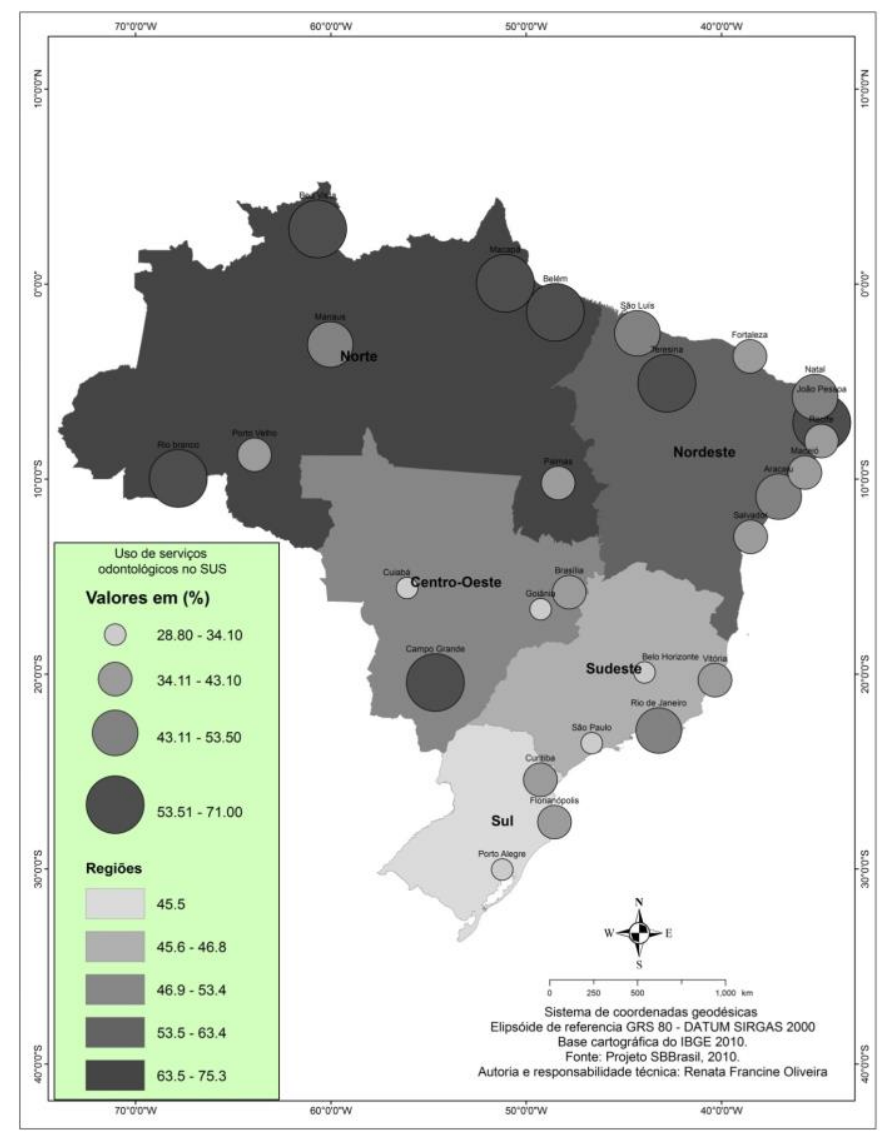

Fonte: Dados próprios

Considerando o nível individual, a média de idade foi de 16,86 anos $( \pm 1,46)$ e a escolaridade média foi de $9,26( \pm 2,41)$ anos de estudo. Houve um predomínio de pessoas de sexo feminino $(52,4 \%)$, que se autodeclararam negros/pardos/amarelos/indígenas (54,4\%) e com renda familiar menor que $\mathrm{R} \$$ $1500,00(66,2 \%)$. Dentre as opções de uso de serviços odontológicos, o SUS foi apontado pela maioria $(53,2 \%)$, como sendo o local da sua última consulta odontológica. Em relação ao tempo decorrido desde esta consulta, 54,7\% relataram que a fizeram no último ano $(54,7 \%)$, predominantemente por motivo de dor/tratamento/extração/outros (64,2\%). (Tabela 1). 
Tabela 1 - Análise descritiva e bivariada dos fatores associados ao uso de serviços odontológicos provenientes do SUS entre adolescentes brasileiros, $n=4654$

\begin{tabular}{|c|c|c|c|c|c|}
\hline \multirow{2}{*}{\begin{tabular}{l}
\multicolumn{1}{c}{ VARIÁVEIS } \\
$\begin{array}{l}\text { Determinantes Primários } \\
\text { Individuais }\end{array}$ \\
\end{tabular}} & \multirow[b]{2}{*}{ n $\left(\%{ }^{*}\right)$} & \multicolumn{3}{|c|}{$\begin{array}{c}\text { USO SERVIÇOS } \\
\%\end{array}$} & \multirow[b]{2}{*}{ Valor p Chi-2 } \\
\hline & & Deff & Outros & SUS & \\
\hline \multicolumn{6}{|l|}{ Idade (em anos) } \\
\hline$\geq 15<18$ anos & $2898(60,6)$ & 5,21 & 50,4 & 49,6 & \\
\hline$\geq 18<19$ anos & $1756(39,4)$ & 5,21 & 56,1 & 43,9 & 0,000 \\
\hline \multicolumn{6}{|l|}{ Anos de estudo } \\
\hline$>12$ & $700(15,8)$ & 13,19 & 66,7 & 33,3 & \\
\hline $9-11$ & $2332(54,0)$ & 8,30 & 57,6 & 42,4 & \\
\hline $5-8$ & $1463(27,1)$ & 8,76 & 40,1 & 59,9 & \\
\hline $0-4$ & $153(3,2)$ & 8,37 & 30,1 & 69,9 & 0,000 \\
\hline \multicolumn{6}{|l|}{ Renda familiar (em reais) ${ }^{a}$} \\
\hline$\geq 1500,00$ & $1483(33,8)$ & 15,56 & 70,5 & 29,5 & \\
\hline$<1500,00$ & $2898(66,2)$ & 15,56 & 42,3 & 57,7 & 0,000 \\
\hline \multicolumn{6}{|l|}{ Sexo } \\
\hline Feminino & $2553(52,4)$ & 5,90 & 51,6 & 48,4 & \\
\hline Masculino & $2101(47,6)$ & 5,90 & 53,7 & 46,3 & 0,151 \\
\hline \multicolumn{6}{|l|}{ Cor da pele autodeclarada } \\
\hline Brancos & $1928(45,6)$ & & 62,4 & 37,6 & \\
\hline $\begin{array}{l}\text { Negros/Pardos// } \\
\text { Amarelos/Indígenas }\end{array}$ & $2726(54,4)$ & 11,35 & 45,6 & 54,4 & 0,000 \\
\hline Comportamentos em Saúde & n (\%*) & Deff & Outros & SUS & Valor $p$ \\
\hline \multicolumn{6}{|c|}{$\begin{array}{l}\text { Tempo desde a última consulta } \\
\text { (anos) * }\end{array}$} \\
\hline$\leq 1$ & $2702(54,7)$ & 8,99 & 61,7 & 38,3 & \\
\hline$>1$ & $1907(45,3)$ & 8,99 & 40,2 & 59,8 & 0,000 \\
\hline \multicolumn{6}{|l|}{ Motivo da Consulta * } \\
\hline Revisão/prevenção & $1613(35,8)$ & 12,11 & 57,0 & 43,0 & \\
\hline $\begin{array}{l}\text { Dor/tratamento/extração/ } \\
\text { Outros }\end{array}$ & $3016(64,2)$ & 12,11 & 50,5 & 49,5 & 0,000 \\
\hline Condições de Saúde Bucal & n (\%*) & Deff & Outros & SUS & Valor $p$ \\
\hline \multicolumn{6}{|l|}{ Presença de dentes cariados } \\
\hline 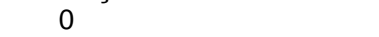 & $2427(47,1)$ & 10,02 & 42,6 & 57,4 & \\
\hline$\geq 1$ & $2167(52,9)$ & 10,02 & 63,9 & 36,1 & 0,000 \\
\hline \multicolumn{6}{|l|}{ Presença de dentes perdidos } \\
\hline $0^{3}$ & $3599(81,0)$ & 6,06 & 55,8 & 44,2 & \\
\hline$\geq 1$ & $995(19,0)$ & 6,06 & 41,0 & 59,0 & 0,000 \\
\hline \multicolumn{6}{|c|}{ Presença de dentes restaurados } \\
\hline 0 & $2091(43,3)$ & 7,75 & 49,9 & 50,1 & \\
\hline \multirow{2}{*}{\multicolumn{6}{|c|}{ Presença de Oclusopatias ${ }^{a}$}} \\
\hline & & & & & \\
\hline Não & $2356(65,1)$ & & 54,3 & 45,7 & \\
\hline \multirow{2}{*}{\multicolumn{6}{|c|}{ Dor de Dente ${ }^{a}$}} \\
\hline & & & & & \\
\hline Não & $3491(74,4)$ & 5,95 & 55,9 & 44,1 & \\
\hline Sim & $1157(25,6)$ & 5,95 & 42,6 & 57,4 & 0,000 \\
\hline \multicolumn{6}{|c|}{$\begin{array}{l}\text { Autopercepção da necessidade } \\
\text { de Tratamento }\end{array}$} \\
\hline Não & $1360(32,2)$ & 11,07 & 66,8 & 33,2 & \\
\hline Sim & $3128(67,8)$ & 11,07 & 46,6 & 53,4 & 0,000 \\
\hline $\begin{array}{l}\text { Autopercepção da Necessid } \\
\text { de Prótese }\end{array}$ & & & & & \\
\hline Não & $4244(95,2)$ & 4,88 & 53,6 & 46,4 & \\
\hline Sim & $264(4,8)$ & 4,88 & 41,3 & 58,7 & 0,000 \\
\hline Satisfação com dentes e boc & & & & & \\
\hline Satisfeito & $2586(56,4)$ & & 58,6 & 41,4 & \\
\hline Indiferente/Insatisfeito & $2035(43,6)$ & 6,22 & 45,2 & 54,8 & 0,000 \\
\hline $\begin{array}{l}\text { Impacto das desordens buce } \\
\text { desempenho diário }\end{array}$ & & & & & \\
\hline Sem impacto & $2902(61,5)$ & 6,13 & 56,3 & 43,7 & \\
\hline Com impacto & $1752(38,5)$ & 6,13 & 46,4 & 53,6 & 0,000 \\
\hline Avaliação do Serviço Usado & & & & & \\
\hline Avaliação da Consulta ${ }^{a}$ & & & & & \\
\hline Muito bom/bom & $3952(85,9)$ & 8,59 & 55,2 & 44,8 & \\
\hline Regular/Ruim/Péssimo & $651(14,1)$ & 8,59 & 38,6 & 61,4 & 0,000 \\
\hline
\end{tabular}

a : n:menor que o total

*: Correção pelo efeito de desenho

Fonte: Dados próprios 
A tabela 1 apresenta os resultados das análises bivariadas, com a distribuição dos adolescentes segundo os fatores individuais em estudo e o tipo de serviço odontológico utilizado.. Nessa análise, os fatores individuais que estiveram relacionados ao uso de serviços odontológicos no SUS com $p<0,20$, foram: idade, anos de estudo, renda familiar, sexo, cor da pele autodeclarada, tempo de uso de serviços odontológicos, motivo do uso dos serviços odontológicos, presença de dentes cariados, presença de dentes perdidos, presença de dentes restaurados, presença de maloclusão, dor de dente, autopercepção da necessidade de tratamento, autopercepção da necessidade de prótese, satisfação com dentes e boca, impacto das desordens bucais no desempenho diário e avaliação da consulta.

Contatou-se que a prevalência do uso de serviços odontológicos no SUS foi maior nas categorias com piores condições sociais e econômicas. Das variáveis contextuais, o menor IDHM e a média de escovação supervisionada abaixo da meta foram associados ao maior uso do SUS. Entre as variáveis individuais, o SUS foi mais usado por aqueles com menor escolaridade, que se autodeclararam negros/pardos/amarelos/indígenas e que haviam utilizados os serviços odontológicos há mais de um ano. Adolescentes cujo motivo de uso dos serviços odontológicos no SUS era dor, tratamentos e extrações usaram mais o SUS. Aqueles que apresentavam dentes cariados, perdidos por cárie, que relataram insatisfação com seus dentes e boca e avaliaram negativamente a consulta odontológica usaram mais o SUS (tabelas 2 e 3 ).

Tabela 2 - Análise de regressão logística multinível dos fatores associados ao uso de serviços odontológicos provenientes do SUS entre adolescentes brasileiros, $n=4654$

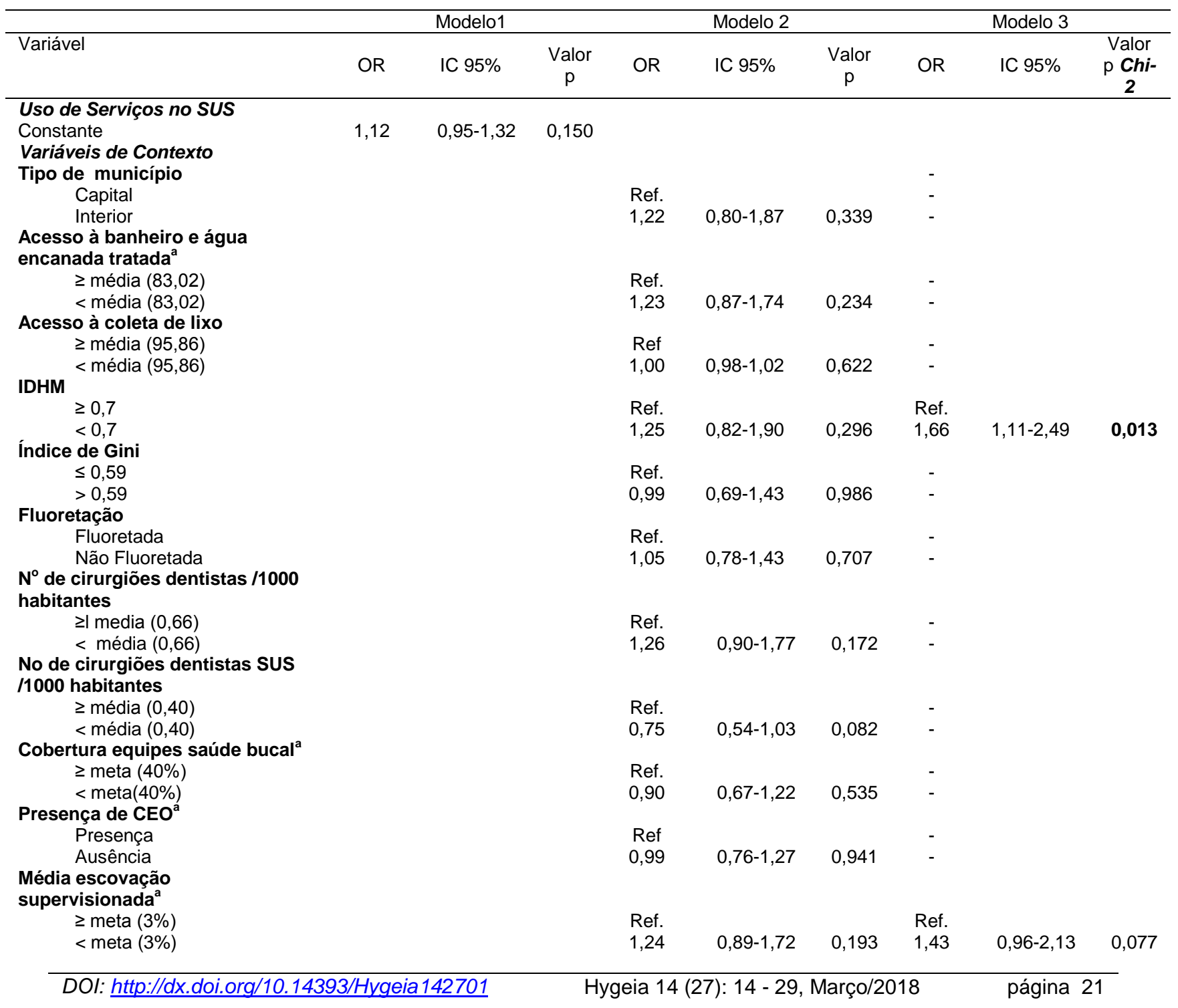


Equidade no uso de serviços odontológicos entre adolescentes brasileiros: uma análise multinível
Renata Francine Oliveira

Desirée Sant'Ana Haikal

Danilo Lima Carreiro

Marise Fagundes Silveira

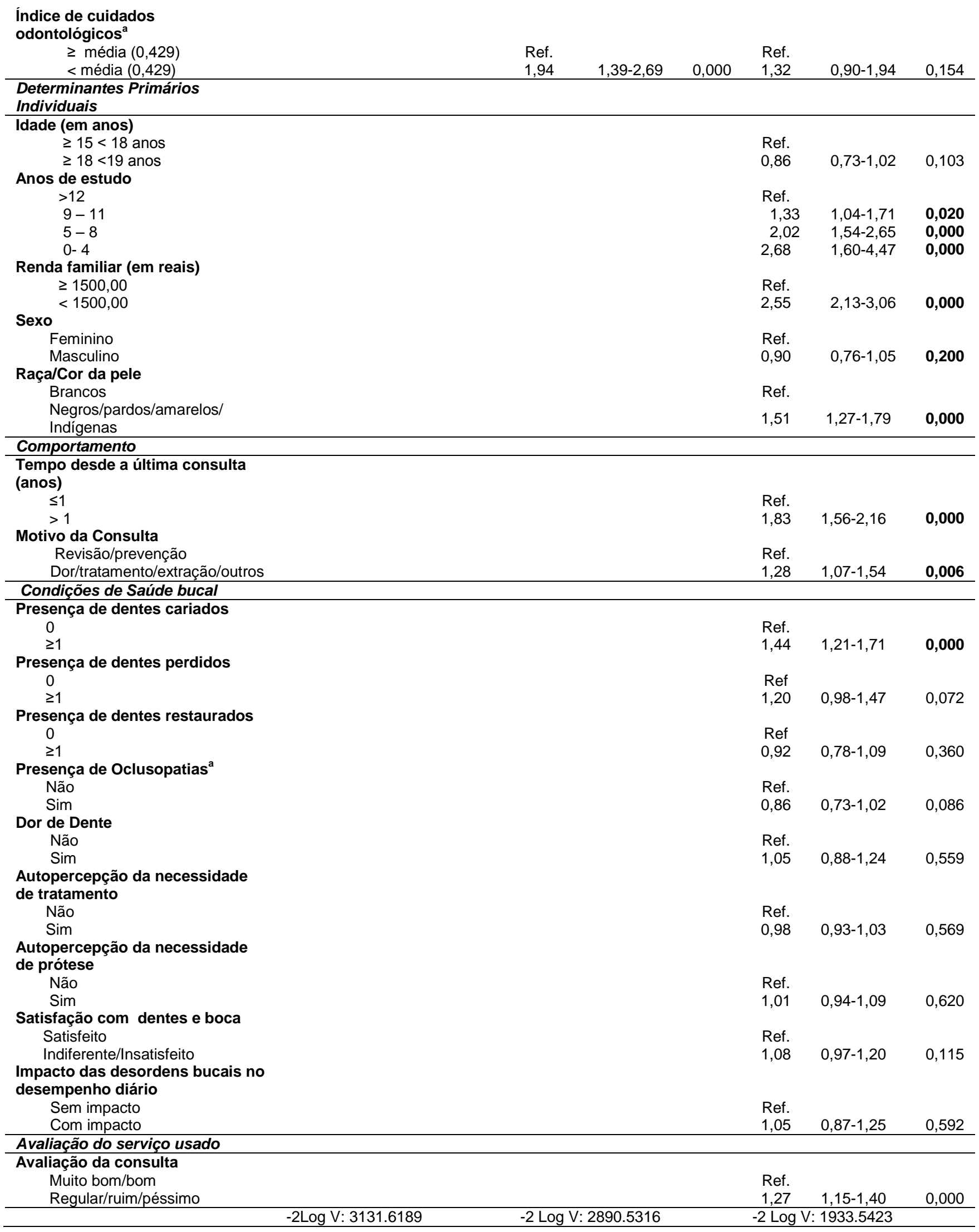

Fonte: Dados próprios 
Tabela 3 - Análise de regressão logística multinível ajustada dos fatores associados ao uso de serviços odontológicos provenientes do SUS entre adolescentes brasileiros, $n=4654$

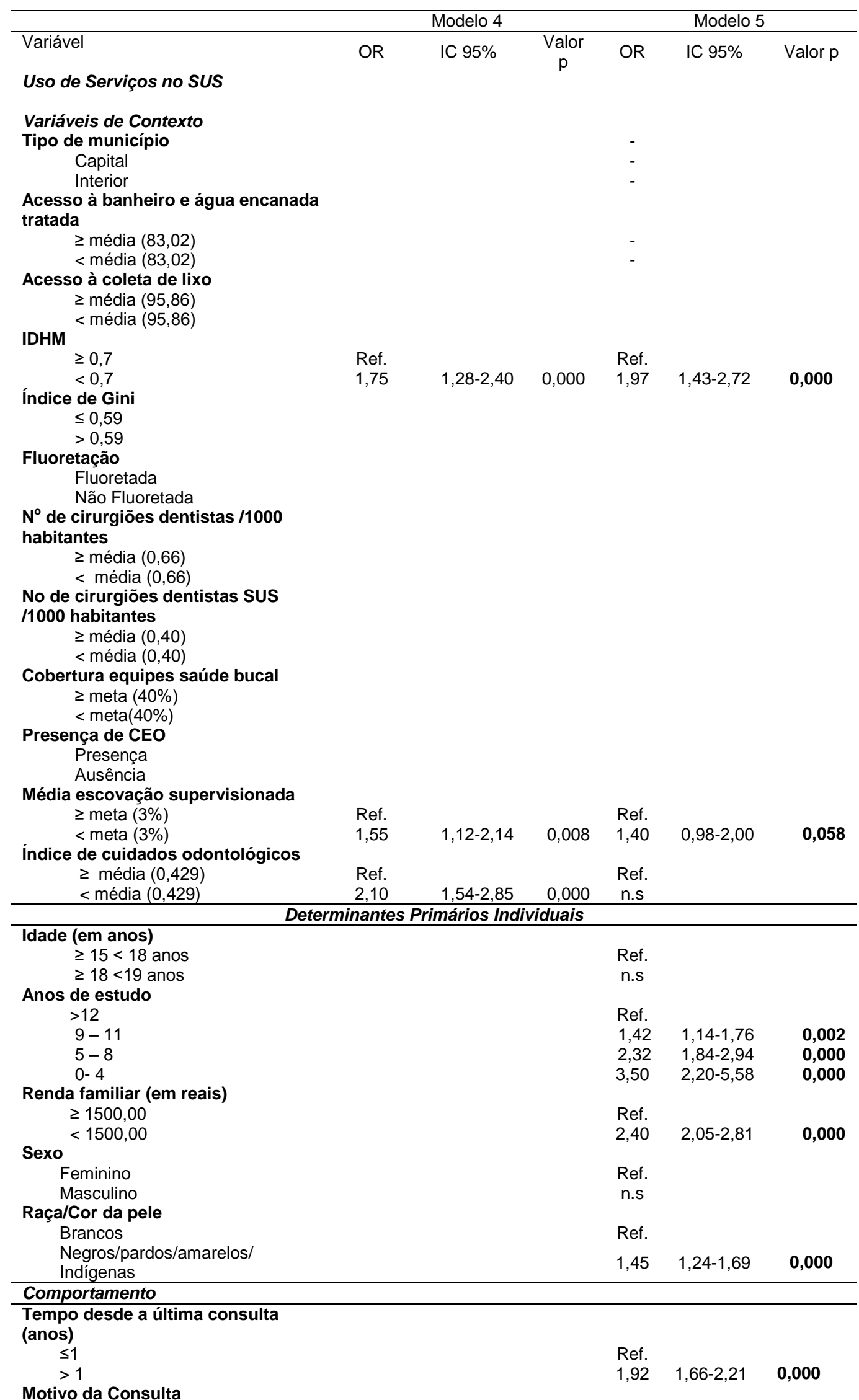




\begin{tabular}{|c|c|c|c|}
\hline $\begin{array}{l}\text { Revisão/prevenção } \\
\text { Dor/tratamento/extração/outros }\end{array}$ & $\begin{array}{l}\text { Ref. } \\
1,33\end{array}$ & $1,13-1,56$ & 0,000 \\
\hline \multicolumn{4}{|l|}{ Condicões de Saúde bucal } \\
\hline \multicolumn{4}{|l|}{ Presença de dentes cariados } \\
\hline 0 & Ref. & & \\
\hline$\geq 1$ & 1,50 & $1,28-1,75$ & 0,000 \\
\hline \multicolumn{4}{|l|}{ Presença de dentes perdidos } \\
\hline 0 & Ref. & & \\
\hline$\geq 1$ & 1,22 & $1,02-1,46$ & 0,026 \\
\hline \multicolumn{4}{|l|}{ Presença de dentes restaurados } \\
\hline 0 & Ref. & & \\
\hline \multirow{2}{*}{\multicolumn{4}{|c|}{ Presença de Oclusopatias ${ }^{a}$}} \\
\hline & & & \\
\hline Não & Ref. & & \\
\hline Sim & n.s & & \\
\hline \multicolumn{4}{|l|}{ Dor de Dente } \\
\hline Não & Ref. & & \\
\hline Sim & n.s & & \\
\hline \multicolumn{4}{|l|}{$\begin{array}{l}\text { Autopercepção da necessidade de } \\
\text { tratamento }\end{array}$} \\
\hline Não & Ref. & & \\
\hline Sim & n.s & & \\
\hline \multicolumn{4}{|l|}{$\begin{array}{l}\text { Autopercepção da necessidade de } \\
\text { prótese }\end{array}$} \\
\hline Não & Ref. & & \\
\hline Sim & n.s & & \\
\hline \multicolumn{4}{|l|}{ Satisfação com dentes e boca } \\
\hline Satisfeito & Ref. & & \\
\hline Indiferente/Insatisfeito & 1,12 & $1,02-1,22$ & 0,009 \\
\hline \multicolumn{4}{|l|}{$\begin{array}{l}\text { Impacto das desordens bucais no } \\
\text { desempenho diário }\end{array}$} \\
\hline Sem impacto & Ref. & & \\
\hline Com impacto & n.s & & \\
\hline \multicolumn{4}{|l|}{ Avaliação do serviço usado } \\
\hline \multicolumn{4}{|l|}{ Avaliação da consulta } \\
\hline Muito bom/bom & Ref. & & \\
\hline Regular/ruim/péssimo & 1,25 & $1,15-1,36$ & 0,000 \\
\hline-2 Log V: 2890.5316 & $-2 \log$ & $\mathrm{V}: 2402.3956$ & \\
\hline
\end{tabular}

Fonte: Dados próprios

\section{DISCUSSÃO}

Este estudo verificou que houve maior proporção de o adolescentes brasileiros que usaram os serviços odontológicos do SUS nos municípios com IDHM mais baixo (médio/baixo/muito baixo) e naqueles que não alcançaram a meta nacional pactuada quanto ao número de escovações supervisionadas,. Em relação às características individuais, foi observado que os adolescentes brasileiros com escolaridade mais baixa, renda familiar menor, que se autodeclararam negros/pardos/amarelos/indigenas, e que buscaram os serviços odontológicos há mais de um ano, também usaram mais os serviços do SUS para cuidados odontológicos. Verificou-se ainda que tais adolescentes usaram mais o SUS para a realização de procedimentos ligados à dor, tratamento e extrações do que para atendimento preventivo/ consulta de rotina. Além disso, os que apresentavam dentes cariados e dentes perdidos, aqueles insatisfeitos com seus dentes e boca e aqueles que avaliaram negativamente a consulta odontológica recebida, também foram maiores usuários do SUS do que de serviços odontológicos de outra natureza.

O tipo de serviço odontológico utilizado pelos adolescentes brasileiros revelou associação com determinantes contextuais e individuais de origem social, biológica, comportamental e relacionada ao serviço, os quais também foram percebidos em outros estudos com amostras locais (Onkuseri, 2013; Frias et al 2007; Travassos, 1997; Paim, 2006).

Ao se avaliar o uso dos serviços odontológicos alguma vez na vida por adolescentes, observa-se, nos inquéritos epidemiológicos nacionais recentes, que ocorreu um decréscimo de apenas $1 \%$ de 2003 para 2010., o que sugere certa estabilidade no acesso dos adolescentes nesse período. O serviço odontológico prestado pelo SUS foi o mais usado pelos adolescentes brasileiros, apesar de ter sofrido um declínio de 2003 para 2010 (Brasil, 2004; 2011). Este achado, no entanto, não deve DOI: http://dx.doi.org/10.14393/Hygeia142701 $\quad$ Hygeia 14 (27): $14-29$, Março/2018 página 24 
necessariamente ser considerado desfavorável, uma vez que, o modelo de atenção do serviço público realizou, neste período, ampliação do acesso a outros grupos etários historicamente excluídos das programações públicas em saúde bucal, como adultos e idosos, e sem que houvesse prejuízos a esse grupo jovem (Brasil, 2006). A proporção de jovens livres de cárie mais que dobrou de um inquérito para o outro. Esse achado sugere que os determinantes da redução da cárie atuaram efetivamente nesta população. As melhorias no poder aquisitivo da população contribuiram nareduçãodas desigualdades e possibilitaram também a ampliaçãodo consumo de serviços privados (Narvai,1999; Marcenes, 200; Nikel et al 2008, Churchi, 1931; Iheozor-Ejiofor et al, 2015; Hoffmann 2009).

Este estudo analisou o padrão de utilização do SUS entre adolescentes brasileiros e verificou que, de uma maneira geral, o princípio da equidade vem sendo alcançado. Ao se falar em equidade, há que se distinguir equidade em saúde de equidade no uso ou no consumo de serviços de saúde, pois segundo Travassos, (1997) os determinantes das desigualdades no adoecer ou morrer são diferentes das desigualdades no consumo de serviços de saúde. As desigualdades em saúde comumente refletem as desigualdades sociais, e a igualdade no uso de serviços de saúde, em função da efetividade das ações de saúde, é condição importante, porém não suficiente, para diminuir as desigualdades existentes entre os grupos sociais.

É importante considerar nos estudos analíticos sobre o uso dos serviços odontológicos, o impacto dos fatores contextuais na definição do uso dos serviços e a partir deste conhecimento, aperfeiçoar os modelos e processo de trabalho propostos no SUS.

Constatou-se, em nível contextual que as variáveis preditoras do uso dos serviços odontológicos no SUS foram: residir em municípios com IDHM com valores muito baixo, baixo e médio $(<0,7)$, estar em municípios que realizam ações coletivas preventivas de escovação dental supervisionada, abaixo da meta definida para o ano de 2010. Percebe-se que estas variáveis se relacionam tanto com a experiência social, que predispõe o adoecer, quanto com o padrão de qualidade e eficiência, existente no serviço público oferecido. O padrão de oferta de ações dos serviços de saúde pode ser de boa ou má qualidade (Fadel, 2009; Brasil 2010). As consequências de uma baixa qualidade dos cuidados prestados recai negativamente sobre a saúde dos pacientes, através da prestação de serviços desnecessários e a execução de procedimentos ineficazes ou insuficientes e até mesmo potencialmente perigosos (Travassos, 1997; Roncalli, 2012; Fadel, 2009).

O IDHM é uma medida resumida das condições básicas de vida de uma população e expressa condições contextuais de vulnerabilidade (Brasil, 2010). Os municípios com IDH alto apresentam para sua população melhores condições de viabilizar o uso dos serviços odontológicos, o que pode representar que, maiores investimentos, financeiros ou estruturais, para viabilizar aumento do acesso aos serviços odontológicos são importantes medidas a serem planejadas.

A partir dos anos 90 , os municípios brasileiros foram estimulados a executarem procedimentos coletivos, dentre eles a escovação supervisionada, para educação e prevenção em saúde bucal (Frazão, 2012; Carvalho, 2009). Os programas de escovação supervisionada, nos quais são utilizados dentifrícios fluoretados, são uma das razões apontadas para o declínio da cárie em escolares brasileiros (Navai, 1999; Frazão, 2012). Segundo Frazão (2012), apesar dos esforços nestas ações serem dirigidos, especialmente para as primeiras séries do ensino fundamental, a análise desta população de adolescentes sugere que a existência de ações preventivas de escovação no município tenha contribuído para a redução na demanda por serviços odontológicos no SUS, devendo ser estimuladas. A organização local e a oferta dos serviços nos municípios impactam diretamente no alcance da equidade. Nos debates sobre esse assunto predominam as discussões relacionadas às questões geográficas de disponibilidade dos serviços bem como à estrutura do serviço local, muitas vezes plural e fragmentada, e provocam um padrão de uso de serviços de saúde iníquo. Sendo assim, as variações existentes entre os diversos sistemas de saúde locais podem reduzir ou ampliar essas desigualdades (Paim, 2006).

No nível individual, as variáveis dos determinantes primários associadas ao uso dos serviços odontológicos no SUS foram a escolaridade, a cor da pele autodeclarada e a renda média familiar. Esses resultados também sugerem que a equidade vem sendo alcançada no SUS. A escolaridade é reconhecidamente um forte preditor de acesso e equidade nos serviços de saúde (Onkuseri, 2006; Frias et al, 2007). A diferença existente em relação à cor da pele indica um favorecimento para brancos em detrimento das outras cores de pele (Onkuseri, 2006). Grupos sociais apresentam demanda diferenciada aos serviços de saúde, tanto no volume quanto no tipo de problema e isso

DOI: http://dx.doi.org/10.14393/Hygeia142701 Hygeia 14 (27): 14 - 29, Março/2018 página 25


precisa ser considerado na programação da oferta de serviços quando se busca equidade (Paim, 2006). Também a renda média familiar exerce influência no padrão de escolha do tipo de serviço odontológico a ser buscado. Diferentemente dos serviços médicos que são, em sua maioria, utilizados pelo SUS, os serviços odontológicos ocorrem com a maior prevalência de serviços privados gerando aumento de gastos nas famílias e ocasionando redução do acesso às famílias mais pobres (Barros \& Bertoldi, 2010). Neste sentido as famílias de baixa renda optam pelos serviços públicos que são gratuitos reafirmando o alcance da equidade.

$\mathrm{Na}$ análise dos comportamentos individuais relacionados ao perfil do serviço de saúde procurado, foram associados ao maior uso do SUS adolescentes que demoravam mais tempo para se consultarem e um maior uso entre aqueles em que o motivo do atendimento foi para dor/tratamento/extração, quando comparados aos que buscaram revisão/prevenção. Comparado com outros grupos etários, os jovens apresentam um perfil mais positivo de comportamentos em saúde, porém, ainda existe um alto nível de demanda de doença acumulada à espera de atendimentos. Em outro estudo ocorreu associação do uso de serviços públicos para pessoas com pior saúde bucal (Gibilini, 2010; Brasil, 2011). A organização dos atendimentos no SUS deve se dar, não pela ordem de procura e sim pela avaliação de risco dos pacientes, que determinará a priorização da atenção àqueles com doença ativa e organizará o intervalo de retornos e manutenções de tratamento (Brasil, 2006).

Piovesan et al (2011) afirmam que o maior uso do SUS para motivos ligados à dor, tratamento e extração pode estar relacionado à autopercepção de saúde decorrente de maior acesso à programas de educação e consequente informação em saúde. Outra possível explicação para esta procura pelo serviço pode ser pela maior facilidade de acesso nesta faixa etária do que nas outras (Matos et al 2002). Quando se compara com outros grupos etários, os jovens apresentam um perfil mais positivo de comportamentos em saúde (Piovesan et al 2011). As razões que levam as pessoas a consultarem um dentista provêm da interação de fatores demográficos, socioeconômicos, psicológicos, e dos perfis de morbidade, sendo que os efeitos e a importância relativa de cada fator são afetados pela bagagem cultural, pelas políticas de saúde vigentes e as características do sistema de saúde (Mendoza-Sassi, et al 2003). A periodicidade em se buscar e alcançar atenção em saúde é muito importante, pois, através dela, acredita-se que os benefícios da intervenção sejam otimizados e reforçados, permite-se o monitoramento do risco, a realização de orientações preventivas e facilita uma atenção mais adequada aos indivíduos em alto risco (Mendoza-Sassi et al, 2003; Freddo et al 2008).

Quanto aos determinantes individuais do uso no SUS relativos às condições de saúde bucal objetivas e subjetivas, foram associados à experiência de cárie com a presença de dentes cariados e perdidos, a satisfação autopercebida com os dentes e a boca e avaliação da consulta odontológica. Em todas essas situações, o uso dos serviços odontológicos do SUS, tiveram associados às condições mais negativas, normativas e subjetivas, corroborando novamente com ideia da importância do SUS para estas pessoas no alcance de maior equidade. Na literatura, o uso de serviços odontológicos em adolescentes e a presença de agravos à saúde bucal também tem forte relação com renda, raça/etnia e escolaridade (Onkuseri, 2006; Frias et al, 2007; Claro et al, 2006; Medina-Solís et al 2013). Apesar de não haver evidências de que o aumento na oferta de serviços cause diminuição das iniquidades, entende-se que ele pode aumentar a possibilidade de acesso, dos grupos menos favorecidos e reduzir as sequelas dos agravos em saúde bucal nessa população (Baldani \& Antunes, 2011). Para que isso ocorra, o desenho do modelo assistencial no nível local é muito importante, pois, fornece a oportunidade maior ou menor, de utilização dos serviços. Tem-se observado no país, uma maior preocupação com aumento de cobertura, aliada a estratégias para garantia de qualidade e fortalecimento das equipes de Atenção Básica em seus atributos essenciais, com um destaque para o acesso (Davoglio et al, 2009).

O modelo teórico adotado comprova a importância das informações contextuais e individuais para auxiliar na compreensão e análise dos determinantes do uso de serviços odontológicos. A abordagem multinível, coerente com a epidemiologia social, reconhece que nem todos os determinantes podem ser conceituados como atributos de nível individual (Guimarães et al, 2011). A inclusão de variáveis contextuais, além das individuais, permitiu captar e evidenciar que o contexto social exerce importante influencia na utilização dos serviços odontológicos no âmbito do SUS.

Uma limitação do estudo é o delineamento transversal, que impede de se inferir causalidade, pela ausência de avaliação temporal. Além disso, é também uma limitação a ser considerada a utilização DOI: http://dx.doi.org/10.14393/Hygeia142701 Hygeia 14 (27): 14 - 29, Março/2018 página 26 
de dados ecológicos. Embora tenham sido utilizadas apenas bases de dados secundários oficiais, ainda assim, há o risco de perda da qualidade da informação coletada e/ou problemas na digitação/alimentação dos dados, com possibilidade de apresentar problemas de confiabilidade e validade (Alves et al, 2014).

A universalidade do acesso e a redução das desigualdades sociais são condições fundamentais para o aumento da equidade (Solar \& Irwin, 2007). No SUS, a gratuidade dos serviços evita que barreiras econômicas restrinjam o consumo, porém outros gastos referentes a transporte e aquisição de medicamento podem também representar barreiras para os grupos de menor renda e que geralmente vivem em áreas onde a disponibilidade de serviços é menor, dificultando o acesso (Travassos, 1997). Os planejamentos em saúde precisam comtemplar as necessidades em saúde de cada população em seu contexto. Assim, o planejamento em saúde que prime por uma ação territorializada traz em seu bojo o enfrentamento das desigualdades sociais que potencializam e nutrem as iniquidades em saúde.

A maior utilização dos serviços de saúde bucal no âmbito do SUS demonstra que a redução das iniquidades reside no maior e mais efetivo acesso ao direito à saúde por adolescentes. Ainda que se mantenham determinantes sociais de saúde, a presença de serviços públicos de saúde bucal nos cenários mais desfavorecidos revela o enfrentamento possível mais imediato no campo das políticas de saúde no Brasil.

Assim, a presente investigação revelou um panorama otimista no desempenho do SUS, ao se observar que está sendo desempenhado seu papel de promover maior equidade entre adolescentes, embora se reconheça que ainda há muito a ser perseguido no alcance de uma assistência odontológica realmente universal e integral no âmbito dos serviços públicos. Ao observar que este serviço tem sido predominantemente utilizado por adolescentes em maior condição de desvantagem e vulnerabilidade contextual e individual, acredita-se que o primeiro passo a ser conquistado em busca da maior equidade em saúde bucal esteja sendo dado.

\section{REFERÊNCIAS}

ALVES, Márcia Guimarães de Mello et al. Fatores condicionantes para o acesso às equipes da Estratégia Saúde da Família no Brasil. Saúde debate, v. 38, n. spe, p. 34-51, 2014.

BALDANI, Márcia Helena; ANTUNES, José Leopoldo Ferreira. Inequalities in access and utilization of dental services: a cross-sectional study in an area covered by the Family Health Strategy. Cadernos de Saúde Pública, v. 27, p. s272-s283, 2011. https://doi.org/10.1590/S0102-311X2011001400014

BARROS, Aluísio JD; BERTOLDI, Andréa D. Desigualdades na utilização e no acesso a serviços odontológicos: uma avaliação em nível nacional. Ciênc saúde coletiva, v. 7, n. 4, p. 709-17, 2002.

BASTOSI, João Luiz; BARROSII, Aluisio JD. Redução das desigualdades sociais na utilização de serviços odontológicos no Brasil entre 1998 e 2008.Rev saúde pública, v. 46, n. 2, p. 250-8, 2012. https://doi.org/10.1590/S0034-89102012000200007

BORGES, Carolina Marques; PERES, Marco Aurélio; PERES, Karen Glazer. Associação entre presença de oclusopatias e insatisfação com a aparência dos dentes e gengivas: estudo com adolescentes brasileiros. Revista Brasileira de Epidemiologia, v. 13, p. 713-723, 2010. https://doi.org/10.1590/S1415-790X2010000400015

BRASIL. Lei no 8.080, de 19 de setembro de 1990: Dispõe sobre as condições para a promoção, proteção e recuperação da saúde, a organização e o funcionamento dos serviços correspondentes e dá outras providências. Diário Oficial da união, v. 128, n. 182, 1990.

BRASIL. Ministério da Saúde. Secretaria de Atenção à Saúde. Departamento de Atenção Básica. Coordenação Nacional de Saúde Bucal. Projeto SB Brasil 2003: condições de saúde bucal da população brasileira 2002-2003-resultados principais. Brasília: MS, 2004.

BRASIL. Ministério da Saúde. A experiência brasileira em sistemas de informação em saúde. Ministério da Saúde, Organização Pan-Americana da Saúde, Fundação Oswaldo Cruz. - Brasília : Editora do Ministério da Saúde, 2009. 
BRASIL. Ministério da Saúde. Portaria no 3.840. Inclui a Saúde Bucal no Monitoramento e Avaliação do Pacto pela Saúde, e estabelece as diretrizes, orientações e prazos do processo de ajuste de metas para o ano de 2011. Diário Oficial da União. 2010.

BRASIL, A. Atlas do desenvolvimento humano no Brasil 2010. Disponível em: http://goo. gl/RMdKjG>. Acesso em, 30/01/2016.

BRASIL. Ministério da Saúde. Secretaria de Atenção à Saúde. Departamento de Atenção Básica. Coordenação Nacional de Saúde Bucal. Projeto SB Brasil 2010: Condições de Saúde Bucal da População Brasileira, Resultados Principais. Brasília: MS, 2011.

CARVALHO, Luís Antonio Cherubini et al. Procedimentos coletivos de saúde bucal: gênese, apogeu e ocaso. Saúde e Sociedade, v. 18, n. 3, p. 490-499, 2009.

CADERNOS DE ATENÇÃO BÁSICA. Saúde Bucal. Brasília-DF: Editora Ministério da Saúde, 2006.

CHURCHILL, H. V. Occurrence of fluorides in some waters of the United States. Industrial \& Engineering Chemistry, v. 23, n. 9, p. 996-998, 1931. https://doi.org/10.1021/ie50261a007

CLARO, Lenita Barreto Lorena et al. Adolescentes e suas relações com serviços. Cad. saúde pública, v. 22, n. 8, p. 1565-1574, 2006.

COSTA, José Felipe Riani; CHAGAS, Luciana de Deus; SILVESTRE, Rosa Maria. A política nacional de saúde bucal do Brasil: registro de uma conquista histórica. In: Técnica. Desenvolvimento de Sistemas e Serviços de Saúde. OPAS, 2006.

DAVIDSON, P. L.; ANDERSEN, R. M. Determinants of dental care utilization for diverse ethnic and age groups. Advances in Dental Research, v. 11, n. 2, p. 254-262, 1997. https://doi.org/10.1177/08959374970110020801

DAVOGLIO, Rosane Silvia et al. Fatores associados a hábitos de saúde bucal e utilização de serviços odontológicos entre adolescentes. Cadernos de Saúde Pública, v. 25, n. 3, p. 655-667, 2009.

DE MATTOS, Ruben Araujo. Princípios do Sistema Único de Saúde (SUS) e a humanização das práticas de saúde. Interface-Comunicação, Saúde, Educação, v. 13, n. 1, p. 771-780, 2009.

DONABEDIAN, Avedis. The quality of care: How can it be assessed?. Jama, v. 260, n. 12, p. 17431748, 1988. https://doi.org/10.1001/jama.1988.03410120089033

FADEL, Marianella Aguilar Ventura; REGIS FILHO, Gilsée Ivan. Percepção da qualidade em serviços públicos de saúde: um estudo de caso. Revista de Administração Pública, v. 43, n. 1, p. 7-22, 2009. https://doi.org/10.1590/S0034-76122009000100002

FRAZÃO, Paulo. Custo-efetividade da escovacao dental supervisionada convencional e modificada na prevencao da carie em molares permanentes de criancas de 5 anos de idade. Cad. Saúde Pública, v. 28, n. 2, p. 281-290, 2012. https://doi.org/10.1590/S0102-311X2012000200007

FREDDO, Silvia Letícia et al. Hábitos de higiene bucal e utilização de serviços odontológicos em escolares de uma cidade da Região Sul do Brasil.Cadernos de saúde pública. Rio de Janeiro. Vol. 24, n. 9 (set. 2008), p. 1991-2000, 2008.

FRIAS, Antônio Carlos et al. Determinantes individuais e contextuais da prevalência de cárie dentária não tratada no Brasil. Rev Panam Salud Publica, v. 22, n. 4, p. 279-85, 2007.

GIBILINI, Cristina et al. Acesso a serviços odontológicos e auto-percepção da saúde bucal em adolescentes, adultos e idosos. Arquivos em odontologia, v. 46, n. 4, p. 213-223, 2010.

GIFT, H. C.; ATCHISON, K. A.; DRURY, T. F. Perceptions of the natural dentition in the context of multiple variables. Journal of dental research, v. 77, n. 7, p. 1529-1538, 1998.

https://doi.org/10.1177/00220345980770070801

GUIMARÃES, Maria Aparecida Martins Baêta et al. Associação entre as ações de serviços de saúde bucal, Índice de Desenvolvimento Humano, presença de água fluoretada e cárie dentária em crianças aos cinco anos de idade. Arquivos em Odontologia, v. 47, n. 3, p. 135-145, 2011.

HOFFMANN, Rodolfo. Desigualdade da renda e das despesas per capita no Brasil, em 2002-2003 e 2008-2009, e avaliação do grau de progressividade ou regressividade de parcelas da renda familiar. Economia e sociedade, v. 19, n. 3, p. 647-661, 2016. 
IBGE. Instituto Brasileiro de Geografia e Estatística. Síntese de indicadores sociais: uma análise das condições de vida da população brasileira. Estudos e Pesquisas. 2011;27:1-4.

IHEOZOR-EJIOFOR, Zipporah et al. Water fluoridation for the prevention of dental caries. The Cochrane Library, n. 6, 2015.

MACHADO, M. F. A. S. et al. Integralidade, formação de saúde, educação em saúde e as propostas do SUS: uma revisão conceitual. Ciênc saúde coletiva, v. 12, n. 2, p. 335-42, 2007.

MARCENES, Wagner; FREYSLEBEN, Gloria Ribeiro; AURELIO DE ANSELMO PERES, Marco. Contribution of changing diagnostic criteria toward reduction of caries between 1971 and 1997 in children attending the same school in Florianopolis, Brazil. Community dentistry and oral epidemiology, v. 29, n. 6, p. 449-455, 2001. https://doi.org/10.1034/j.1600-0528.2001.290606.x

MATOS, Divane Leite et al. Projeto Bambuí: avaliação de serviços odontológicos privados, públicos e de sindicato. Revista de Saúde Pública, v. 36, n. 2, p. 237-243, 2002.

MEDINA-SOLÍS, C. E. et al. Treatment needs for Dental Caries, Restorative Care Index, and Index of Extractions in adolescents 12 and 15 years old.West Indian Medical Journal, v. 62, n. 7, p. 636-641, 2013.

MENDOZA-SASSI, Raúl; BÉRIA, Jorge U.; BARROS, Aluísio JD. Outpatient health service utilization and associated factors: a population-based study.Revista de saúde pública, v. 37, n. 3, p. 372-378, 2003. https://doi.org/10.1590/S0034-89102003000300017

NARVAI, Paulo C.; FRAZÃO, Paulo; CASTELLANOS, Roberto A. Declínio na experiência de cárie em dentes permanentes de escolares brasileiros no final do século XX. Odontologia e sociedade, v. 1 , n. 1/2, p. 25-29, 1999.

NICKEL, Daniela Alba; LIMA, Fábio Garcia; DA SILVA, Beatriz Bidigaray. Modelos assistenciais em saúde bucal no Brasil Dental care models in Brazil. Cad. Saúde Pública, v. 24, n. 2, p. 241-246, 2008.

PAIM, Jairnilson Silva. Eqüidade e reforma em sistemas de serviços de saúde: o caso do SUS. Saúde e Sociedade, v. 15, n. 2, p. 34-46, 2006. https://doi.org/10.1590/S010412902006000200005

PAIM, Jairnilson et al. The Brazilian health system: history, advances, and challenges. The Lancet, v. 377, n. 9779, p. 1778-1797, 2011.

PAVÃO, Ana Luiza Braz; COELI, Cláudia Medina. Modelos teóricos do uso de serviços de saúde: conceitos e revisão. Cad. saúde colet.,(Rio J.), v. 16, n. 3, 2008.

PERES, Marco A. et al. Desigualdades no acesso e na utilização de serviços odontológicos no Brasil: análise do Sistema de Vigilância de Fatores de Risco e Proteção para Doenças Crônicas por Inquérito Telefônico (VIGITEL 2009). Cad Saúde Pública, v. 28, n. sSuppl, 2012.

PIOVESAN, Chaiana et al. Influence of self-perceived oral health and socioeconomic predictors on the utilization of dental care services by schoolchildren. Brazilian oral research, v. 25, n. 2, p. 143-149, 2011.

RONCALLI, Angelo Giuseppe et al. Aspectos metodológicos do Projeto SBBrasil 2010 de interesse para inquéritos nacionais de saúde. Cad. saúde pública, v. 28, n. supl, p. s40-s57, 2012.

SNIDJERS, T. A. Multilevel analysis Springer Berlin Heidelberg.2011; 879-882.

SOLAR, Orielle; IRWIN, Alec. A conceptual framework for action on the social determinants of health. 2007.

SUSSEX, P. Most Cited: number 10. International patterns of oral health care--the example of New Zealand. The New Zealand dental journal, v. 106, n. 3, p. 115-116, 2010.

OKUNSERI, Christopher et al. Predictors of dental care use: findings from the national longitudinal study of adolescent health. Journal of Adolescent Health, v. 53, n. 5, p. 663-670, 2013. https://doi.org/10.1016/.j.jadohealth.2013.05.013

WORLD HEALTH ORGANIZATION et al. The world health report: health systems financing: the path to universal coverage. 2010.

TRAVASSOS, Claudia Maria de Rezende et al. Equidade e o Sistema Único de Saúde: uma contribuição para debate. 1997. 